\title{
Summary of the National Consensus Conference on Foodborne, Waterborne and Enteric Disease Surveillance
}

\author{
November 28 to 30, 1995, Citadel Hotel and Convention Centre, \\ Ottawa, Ontario
}

\author{
JefF Wilson PhD, PAul SOCKett PhD, JOHn SPIKA MD, Wendy JOHNSON PhD
}

$\mathrm{T}$ he National Consensus Conference on Foodborne, Waterborne and Enteric Disease Surveillance, held in Ottawa, November 28 to 30,1995 was convened by the Bureau of Infectious Diseases, Laboratory Centre for Disease Control (LCDC), Health Protection Branch (HPB) of Health Canada and chaired by Dr Monique Douville-Fradet, Ministère de la Santé et de Services Sociaux, Québec. The conference was designed to solicit insights and ideas from a representative group of experts and stakeholders as a key source of input on HPB's current Re-Engineering Action Plan, which is focusing on means to strengthen Canada's foodborne, waterborne and enteric disease surveillance system and capabilities.

The Re-Engineering Action Plan will be proposing investments into key population health intelligence network initiatives that will help reduce the risk and burden of foodborne, waterborne and enteric diseases by enabling more timely and effective detection and analysis of these diseases, and of related risk factors. This is a cooperative venture led by LCDC that will engage the support and commitment of other key federal agencies, most notably Agriculture and Agri-Food Canada, Fisheries and Oceans Canada and Environment Canada, provincial and territorial ministries of health and agriculture and food, and other participants in the national system, including the national network of laboratories, food and water inspectors and health authorities.

Laboratory Centre for Disease Control, Health Protection Branch, Health Canada, Ottawa, Ontario

Correspondence and reprints: Dr Jeff Wilson, Department of Population Medicine, University of Guelph, Guelph, Ontario N1G 2W1. Telephone 519-824-4120, ext 4728, fax 519-763-3117, e-mail jwilson@ovcnet.uoguelph.ca
As an important first step in this plan, a preliminary review of the current foodborne, waterborne and enteric disease surveillance system in Canada was carried out by a private consulting firm. Most notable among its observations was a need to make the system more formal and cohesive and, in particular, to bring together the microbiological, epidemiological, regulatory, disease control and clinical elements through more effective communication and partnerships. In recognition of the diversity of participants at the local, federal, provincial and territorial levels, a need was identified to clarify roles, as well as protocols and procedures for gathering, analyzing and disseminating data on disease and risk factors. A need to invest in data management and communication systems to enhance the timeliness of analysis and dissemination was also identified. The existing network of surveillance partners provides a solid base on which to build a more effective foodborne, waterborne and enteric disease surveillance system, and there is considerable good will among key participants to collaborate on improvements.

\section{THE CONSENSUS CONFERENCE}

About 70 participants, spanning a broad range of interests from all levels of government, academic and research institutions, industry and public interest organizations concerned with health issues, attended the Conference. Formal presentations by invited experts from Canada, the United States and Europe were followed by a series of small working group and full plenary sessions that addressed the following issues.

- objectives of foodborne, waterborne and enteric disease surveillance at national, regional and local levels;

- means to enhance the timeliness and usefulness of 
surveillance data, and ideal timelines for data collection, analysis and dissemination;

- mechanisms to better coordinate Canadian foodborne, waterborne and enteric disease surveillance activities;

- priority topics for targeted special studies on foodborne, waterborne and enteric diseases and related risk factors;

- organisms and microbial toxins that should be tested for as part of a national foodborne, waterborne and enteric disease surveillance strategy;

- minimum data that should accompany laboratory-confirmed isolates in reports to national reference laboratories;

- $\quad$ suggested approaches to harmonize laboratory methods and quality assurance programs related to foodborne, waterborne and enteric disease surveillance activity;

- gaps in data collection and transmission that need to be addressed to ensure effective and timely foodborne, waterborne and enteric disease surveillance;

- potential incentives and mechanisms to encourage and promote the collection and transmission of required surveillance data;

- issues related to patient and proprietary confidentiality that affect access to, or use of, foodborne, waterborne and enteric disease surveillance data;

- means to ensure compatibility of software for surveillance networks across Canada;

- required analysis and aggregation of surveillance data, including roles and responsibilities at different levels in the national system;

- content and format of dissemination products and vehicles of foodborne, waterborne and enteric disease surveillance data;

- means to enhance access to surveillance data;

- required surveillance data on determinates and outcome of foodborne, waterborne and enteric disease;

- requirements for national notification of disease outbreaks, by selected pathogens;

- requirements for nationally uniform protocols for investigation of foodborne, waterborne and enteric disease outbreaks.

\section{KEY FINDINGS AND RECOMMENDATIONS}

In total, more than 60 recommendations were proposed, all with the consensual support of the conference participants. Some of the most notable recommendations from the consensus conference follow.

Scope of foodborne, waterborne and enteric disease surveillance: The conference endorsed a broad range of foodborne, waterborne and enteric disease for inclusion in surveillance activities. A number of priority topics for special studies that would greatly enhance the understanding of foodborne, waterborne and enteric diseases and related risk factors were also suggested, in addition to the kinds of surveillance data that should be gathered at local, provincial, territorial and federal levels to ensure adequate and timely detection, analysis and response.

Planning and coordination mechanisms: The conference advocated the creation by LCDC of a national expert body on foodborne, waterborne and enteric disease surveillance having representation from key federal and provincial agencies, laboratories and a variety of public health, industry, academic and consumer interest organizations.

This expert body would be charged with considering specific means by which Canada's foodborne, waterborne and enteric disease surveillance system can be enhanced. It would begin with detailed consideration of most of the recommendations from the consensus conference and the development of recommendations for consideration by the key participants in the system. The expert body's suggested mandate would include formulating recommendations on the structure and coordination of the national surveillance system, mechanisms for improved communication and coordination, approaches to a national surveillance strategy (including processes to secure national consensus on surveillance priorities and approaches), harmonized approaches to data management, and coordinated methods for data access and dissemination.

Laboratory testing and capabilities: The conference outlined the key organisms and microbial toxins that should be regularly tested for as part of a national surveillance strategy for foodborne, waterborne and enteric disease. It identified the organisms and toxins in clinical, food and water samples for which each province and territory should have full testing capability (either in-house or within ready access). In addition, the conference suggested pathogens and toxins that should be the subject of minimum routine laboratory testing capability in each province and territory. Finally, the conference outlined the spectrum of laboratory testing capabilities that should be maintained or developed in Canada for specialized pathogen typing.

Data collection, analysis, access and dissemination: The conference identified specific gaps in surveillance data currently being collected and recommended priority areas for data collection at each level, all based on the principle that surveillance should be selective, focusing on key factors and data most pertinent to early detection and analysis. Much of the detail is required at the local level. The conference also suggested a number of ways by which responsible authorities might be encouraged and assisted in collecting required data, and ensuring their timely dissemination and ready access by others with responsibilities related to foodborne, waterborne and enteric diseases. Considerable emphasis was placed on the importance of rapid updating of data and ready access by authorized users; in this regard, the conference highlighted the need for better, and more compatible, software systems to enhance participation of all members in the national system. 


\section{FOLLOW-UP}

The results of the consensus conference, including highlights of the discussion and complete test of all recommendations, have been incorporated into a conference report that will go to all participants, in addition to key agencies and institutions with active interests in foodborne, waterborne and enteric disease surveillance. Work has begun on establishing the proposed expert body and developing its initial mandate. In the meantime, other initiatives are being pursued to respond to the specific foodborne, waterborne and enteric disease surveillance priorities identified at the consensus conference and in the earlier Re-Engineering Action Plan.

Note: Copies of the Consensus Conference report may be obtained by contacting Ms Nicole Armstrong, Bureau of Infectious Diseases, LCDC, Tunney's Pasture, PL 0603E1, Ottawa, Ontario K1A OL2. 


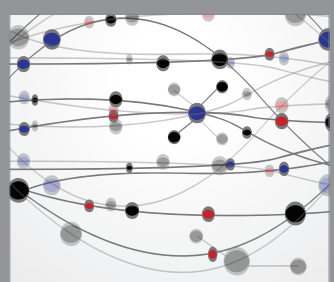

The Scientific World Journal
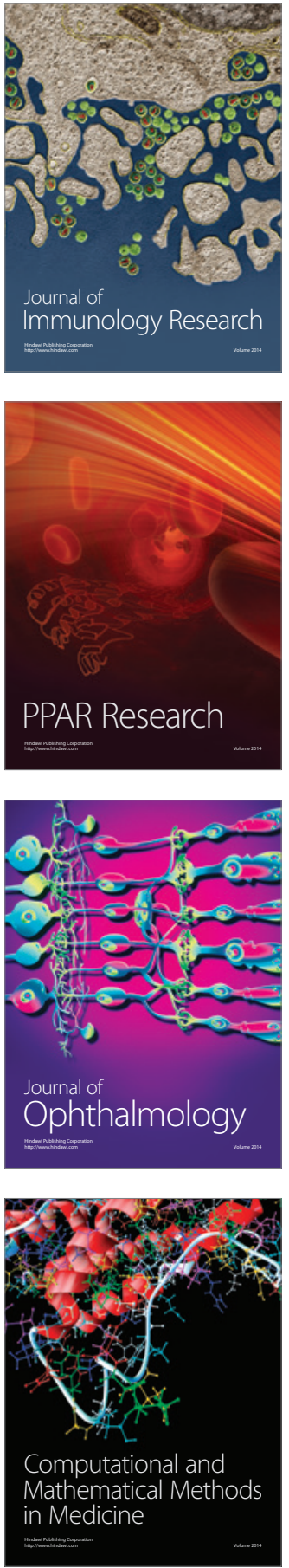

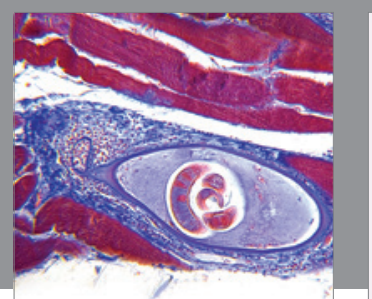

Gastroenterology Research and Practice

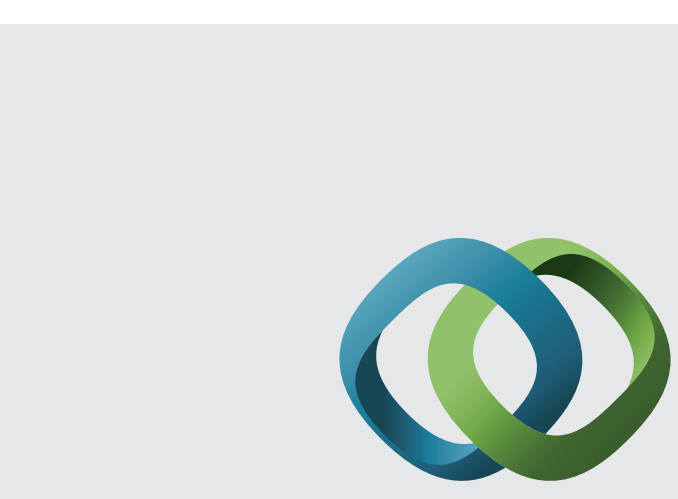

\section{Hindawi}

Submit your manuscripts at

http://www.hindawi.com
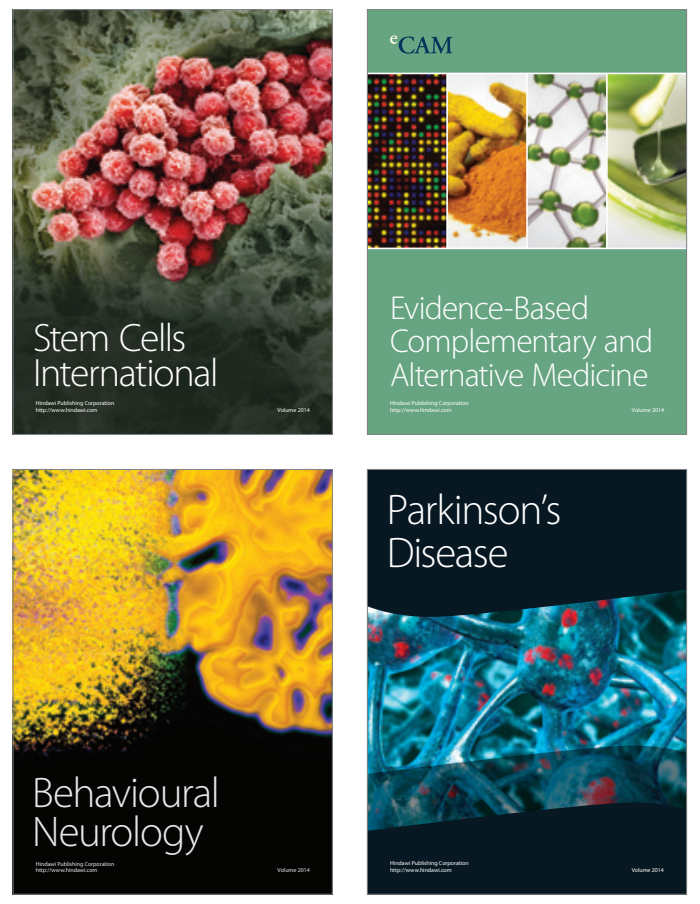
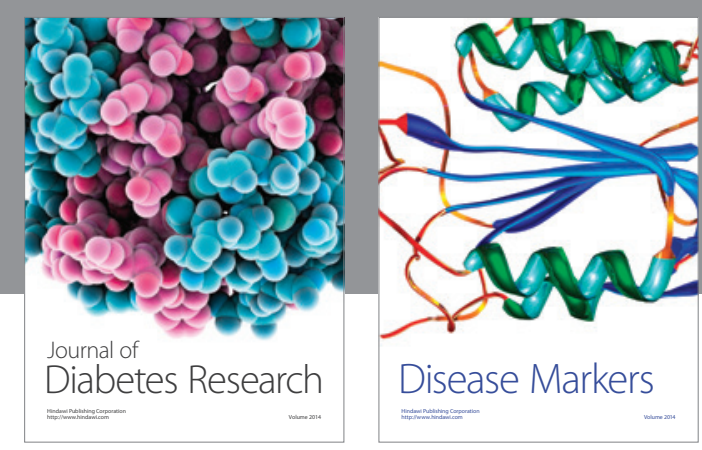

Disease Markers
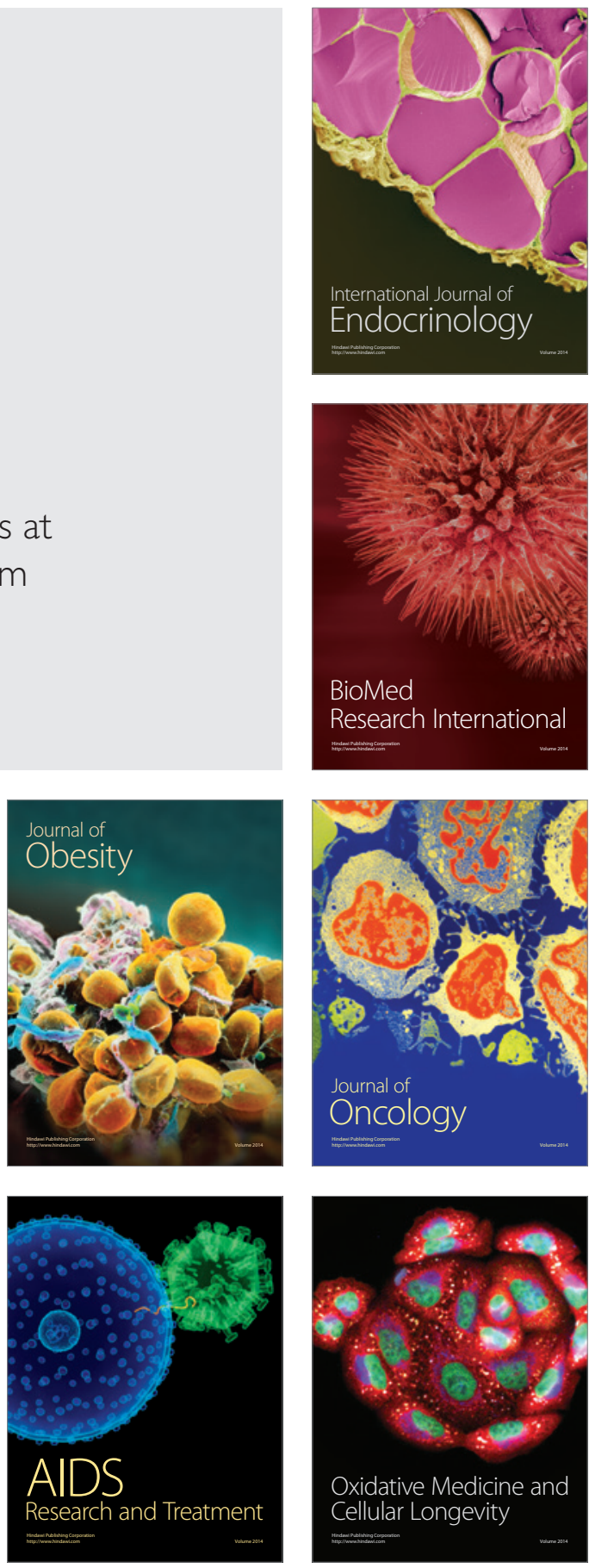